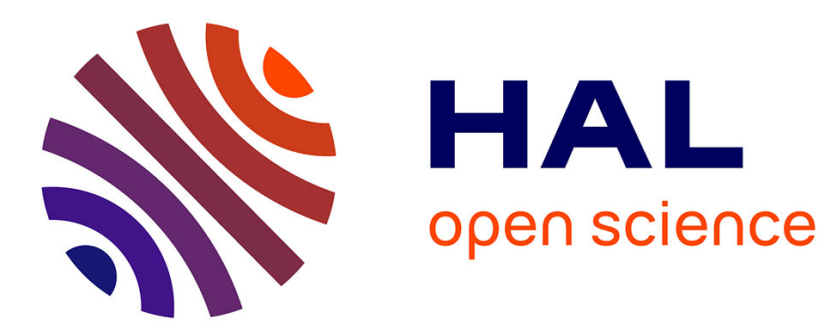

\title{
Simulations using the pulse shape comparison scanning technique on an AGATA segmented HPGe gamma-ray detector
}

\author{
B. de Canditiis, G. Duchêne
}

\section{- To cite this version:}

B. de Canditiis, G. Duchêne. Simulations using the pulse shape comparison scanning technique on an AGATA segmented HPGe gamma-ray detector. Eur.Phys.J.A, 2020, 56 (10), pp.276. 10.1140/epja/s10050-020-00287-6 . hal-03011007

\section{HAL Id: hal-03011007 https://hal.science/hal-03011007}

Submitted on 15 Dec 2020

HAL is a multi-disciplinary open access archive for the deposit and dissemination of scientific research documents, whether they are published or not. The documents may come from teaching and research institutions in France or abroad, or from public or private research centers.
L'archive ouverte pluridisciplinaire HAL, est destinée au dépôt et à la diffusion de documents scientifiques de niveau recherche, publiés ou non, émanant des établissements d'enseignement et de recherche français ou étrangers, des laboratoires publics ou privés. 


\title{
Simulations using the PSCS technique on an S-type AGATA segmented HPGe gamma-ray detector
}

\author{
B. De Canditiis ${ }^{1, a}$, G. Duchêne ${ }^{1}$ \\ ${ }^{1}$ Université de Strasbourg, CNRS, IPHC UMR 7178, F-67000 Strasbourg, France
}

Received: / Accepted:

\begin{abstract}
Monte Carlo simulations are used to test the Pulse Shape Comparison Scanning (PSCS) technique implemented at the IPHC scanning table. The tests, performed on a high purity germanium (HPGe) detector unit of the AGATA array, aim to quantify the accuracy of the system and validate it. The simulations use a combination of tools such as Geant4, SIMION and the Agata Detector Library (ADL). Quality parameters are extracted at various gamma-ray energies and the impact of the input statistics on the parameters is also analyzed.
\end{abstract}

Keywords HPGe detectors · Full volume characterization - Pulse Shape Analysis (PSA) · AGATA . Scanning table

\section{Introduction}

The Advanced GAmma Tracking Array (AGATA) [1] is an array of high purity germanium (HPGe) segmented detectors designed and built in the framework of an international collaboration which involves 11 European countries. With its 180 detectors, 41 of which are already in operation in GANIL, the array will cover a solid angle of $\sim 80 \%$ of $4 \pi$. Since 2010 , the incomplete array is used in experiments to study the structure of atomic nuclei as a function of angular momentum, isospin, and temperature.

The 36-fold segmentation of the AGATA detector units makes them position sensitive, as for a given interaction position an uniquely-shaped pulse is generated by the detector. This characteristic leads to the main feature of AGATA, namely its ability to reconstruct the

a e-mail: bartolomeo.decanditiis@iphc.cnrs.fr history of the interactions of a gamma ray propagating in the array, which is possible thanks to dedicated tracking algorithms [2-6]. This makes obsolete the use of Compton shields, as in the previous generation HPGe detector arrays such as EUROBALL [7, 8] and GAMMASPHERE [9], increasing the geometrical efficiency of the tracking array. Moreover, the reconstruction of the gamma-ray trajectories leads to a substantial improvement of the Doppler correction and therefore of the energy resolution. It also allows the measurement of the linear polarization of gamma rays, which is useful for the determination of the parity of the nuclear states of interest.

In order to reconstruct an event, the tracking algorithm needs as input the positions of each interaction of the gamma ray. These are determined with pulse shape analysis (PSA) algorithms [10-15]. For a given interaction, the algorithm compares the experimental pulse shape with a database of calculated pulse shapes associated with spatial coordinates within the volume of the detector. After PSA and gamma-ray tracking, the spatial resolution obtained is $\sim 5 \mathrm{~mm}$ FWHM for $1 \mathrm{MeV}$ interactions [10, 16, 17].

Presently, databases of pulse shapes are calculated with the AGATA Detector Library (ADL) software [10] leading to good tracking performances, although some characteristics of the detector such as the real impurity distribution and the thickness of the dead layers are not always correctly known and their implementation is subject to uncertainties. A novel approach to database construction is the implementation of full volume characterization of the detectors via three-dimensional scans. These measurements are realized with dedicated tools called scanning tables [18-21].

One of such scanning tables is based at the IPHC Strasbourg [22] and is realized within the framework of 
the AGATA collaboration. This scanning table is designed to perform three-dimensional scans with a good spatial resolution, a large amount of scanned points and short running times. The table implements the Pulse Shape Comparison Scan (PSCS) technique [23] that allows the reconstruction of a database by comparing two datasets of pulses. The two datasets are obtained by performing, with a collimated ${ }^{137} \mathrm{Cs}$ source, two twodimensional scans, in which the detector is oriented in two different directions perpendicular to each other (eg. vertical and horizontal orientations).

The aim of the work presented here is to quantify the characteristics of the PSCS technique implemented by the Strasbourg scanning table [24]. The whole scanning procedure is recreated with Monte Carlo simulations of an S-type AGATA detector unit and the data obtained are analyzed with the same algorithms used for the real measurements.

In the first part of this paper, the AGATA detector unit is briefly described along with the signal generation. Subsequently the PSCS technique and its implementation by the IPHC scanning table is presented. The tools of the simulation and the data treatment are then shown. Finally the results of the simulation are described and interpreted. Conclusions and perspectives are given in the last section.

\section{HPGe segmented AGATA detector unit}

The AGATA detector units (figure 1) [1] are segmented, closed-end, coaxial, n-type HPGe crystals that have a tapered hexagonal geometry with an asymmetric shape. Three slightly different tapered shapes exist labeled as A, B and C. Moreover a fourth totally symmetrically shaped unit, the S-type (in figure 2), exists. Only thee detectors with such shape were built as prototypes, only used in tests, and they are not actually part of the array [25]. The length of each crystal is $90 \mathrm{~mm}$ and the coaxial diameter is $80 \mathrm{~mm}$. The tapering angle of the S-type unit is $10^{\circ}$. The central hole, drilled from the back, has a diameter of $10 \mathrm{~mm}$ and extends up to $13 \mathrm{~mm}$ from the front face of the detector. The 6-fold sectorwise segmentation goes through the middle of each flat hexagonal side. The 6 -fold longitudinal segmentation forms segment rings of $8,13,15,18,18$ and $18 \mathrm{~mm}$ in thickness starting from the front face.

Thus 37 signals are collected from each crystal: 36 segment signals plus 1 total energy signal collected by the central contact (core). Each crystal has an impurity concentration between 0.4 and $1.8 \times 10^{10} \mathrm{~cm}^{-3}$. In particular, the S-type detector, which is used as model for the simulations in this work (S001) has an impurity concentration of $1.5 \times 10^{10} \mathrm{~cm}^{-3}$ at the front and

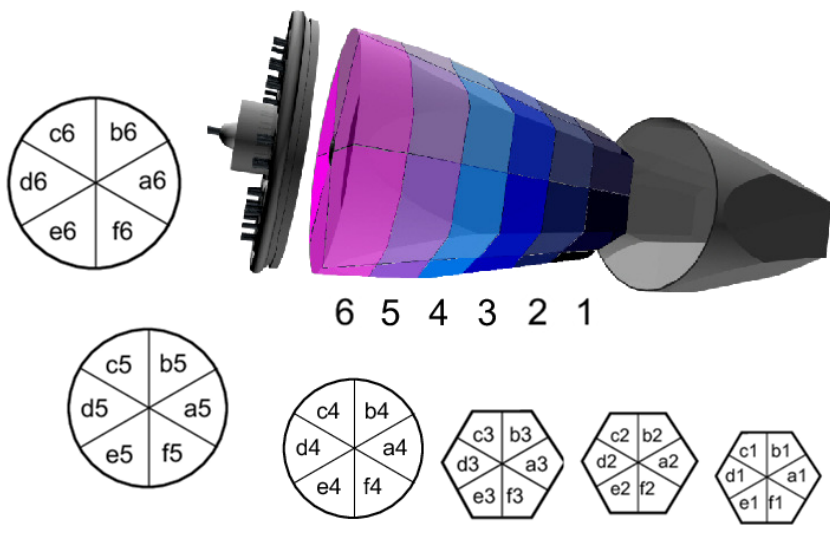

Fig. 1 Exploded rendering of an AGATA detector unit. The six slices of the detector are shown with their respective labeling. The hexagonal shaped slices are towards the front of the detector. The segment labeling corresponds to the detector seen from its back side. Adapted from [1].
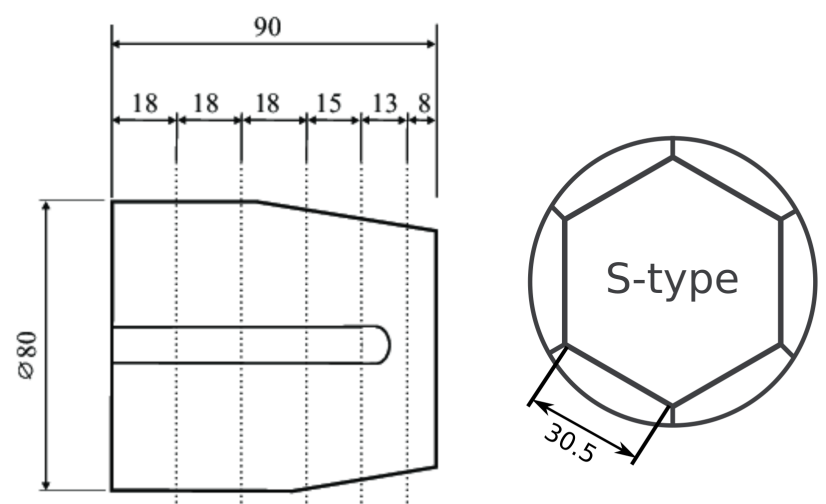

Fig. 2 Dimensions (in mm) of the S-type detector. Adapted from [1].

$0.43 \times 10^{10} \mathrm{~cm}^{-3}$ at the back, as indicated by the manufacturer. The crystals are encapsulated in hermetically sealed aluminum canisters [26] with a $0.8 \mathrm{~mm}$ wall thickness. The spacing between the crystal and the canister wall varies from $0.4 \mathrm{~mm}$ to $0.7 \mathrm{~mm}$.

Details about the embeded cold and warm preamplifier stages are given in [1, 27-29]. Finally the signals generated by the segments and the core for each crystal are sampled with 14 bit ADCs at a $100 \mathrm{MHz}$ rate. This is the case for the AGATA data acquisition [1] as well as for the Strasbourg scanning table experimental setup [22, 30] and will also be used for the simulations.

\subsection{Signal generation}

The shape of the signals generated by a detector can be calculated with the Shockley-Ramo theorem [31-33]. 
The theorem states that the charge $Q$ and the current $i$ on an electrode induced by a moving point-like charge $q$ are given by:

$Q=-q \phi_{0}(\mathbf{x})$

$i=q \mathbf{v} \cdot \mathbf{E}_{0}(\mathbf{x})$

where $\mathbf{v}$ is the instantaneous velocity of the charge $q$. The quantities $\phi_{0}(\mathbf{x})$ and $\mathbf{E}_{0}(\mathbf{x})$ are called weighting potential and weighting field, respectively. They correspond to the electric potential and field that would exist at the instantaneous position $\mathbf{x}$ of the charge $q$ if the selected electrode is considered at $1 \mathrm{~V}$, all the other electrodes being at $0 \mathrm{~V}$, and the charge $q$ removed. As supplementary assumptions, it is considered that the magnetic effects are negligible and that the electric field propagates instantaneously (quasi-static approximation).

The electric field can be calculated analytically or numerically (depending on the complexity of the geometry of the detector) by solving the Poisson equation:

$\triangle \phi=-\frac{\rho}{\epsilon}$

Typically, simple symmetric detector geometries, such as planar or true coaxial configurations, have analytical solutions, while the solution for more complex geometries is generally obtained numerically.

The weighting potentials can be obtained by solving the Laplace equation:

$\nabla^{2} \phi_{i}(\mathbf{x})=0$

where:

$\phi_{i} \mid S_{j}=\delta_{i, j}$

with $S_{j}$ the surface of the electrode $j$. From equations 1 and 2 one can deduce that for a charge $q$, located at position $\mathbf{x}$, and traveling towards the electrode $S_{i}$, this electrode will collect an induced charge $Q$ proportional to the weighting potential $\phi_{i}(\mathbf{x})$. At the same time, the other electrodes $S_{j \neq i}$ will collect an induced charge $Q$ proportional to the value of their respective weighting potential $\phi_{j}(\mathbf{x})$. However, from equation 4 , when $q$ reaches the electrode $S_{i}$ the net charge induced on it will be $Q=-q$, while the net charge collected on the other electrodes will be $Q=0$.

The charge collected on the electrodes $S_{j \neq i}$ is called transient charge (or image charge) and the signals obtained from it are called transient signals (or image signals). Since the weighting potentials strongly depend on the spatial coordinates, the signals generated on the electrodes (especially for the transient signals) can be used to deduce the position of the gamma-ray interaction that generated the charge, making segmented HPGe detectors position sensitive.
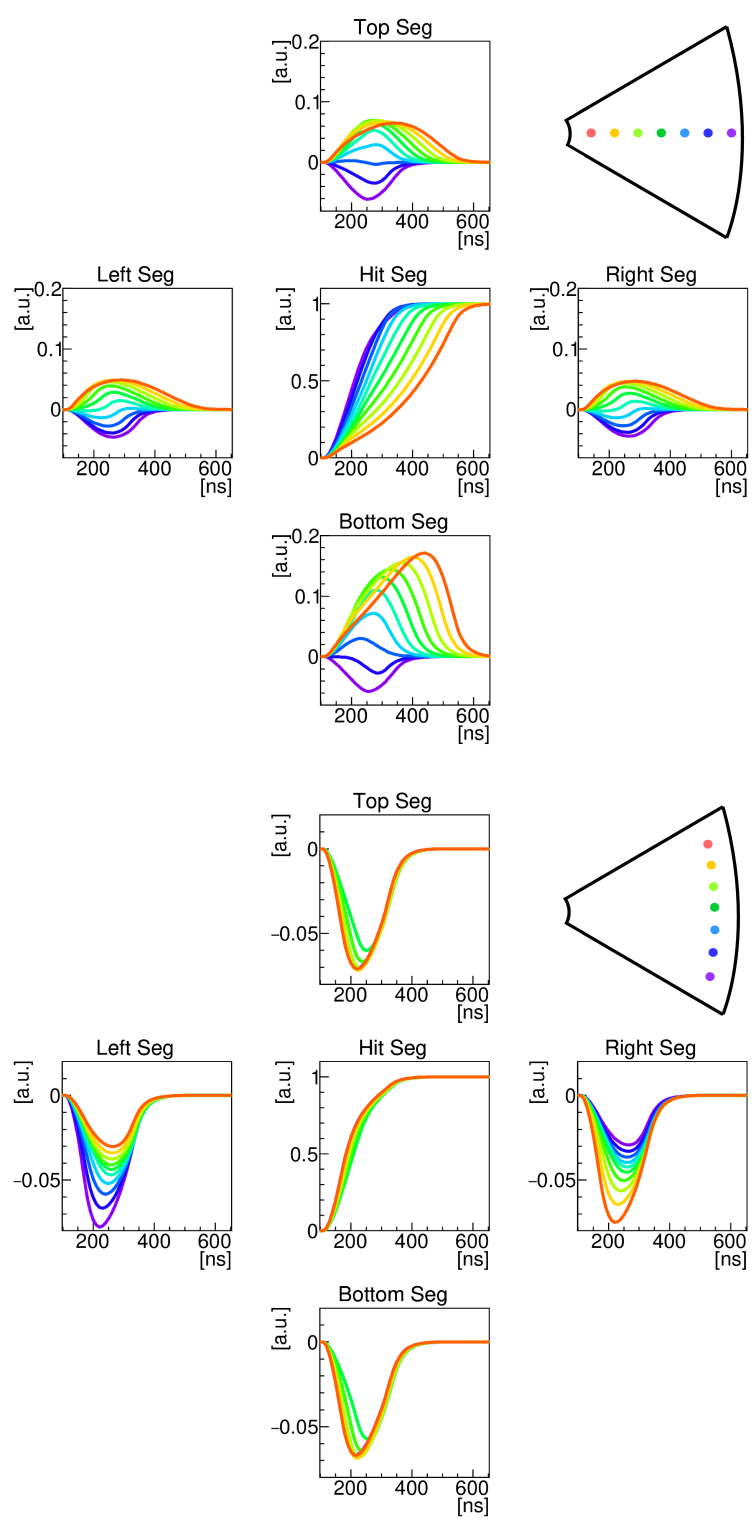

Fig. 3 Pulse shapes calculated with ADL (see section 5) for interactions at different radii (top panels) and at a fixed radius and different lateral positions (bottom panel). For each panel the hit segment (central plot) and the top/bottom and left/right segments are represented. See text for details.

Examples of simulated signals generated by gamma rays interacting in an AGATA detector can be seen in figure 3 . The top figure shows the signals for different interaction radii: the graph in the center shows the main signal generated by the hit segment, while the graphs around show the transient signals of the neighboring segments. The graphs also show the difference in the maximum amplitude between the main signal (normalized to 1) and the transient signals. The figure on the bottom shows the signals for different interactions 


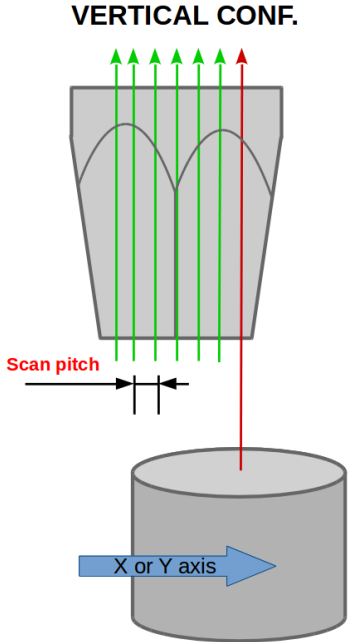

HORIZONTAL CONF

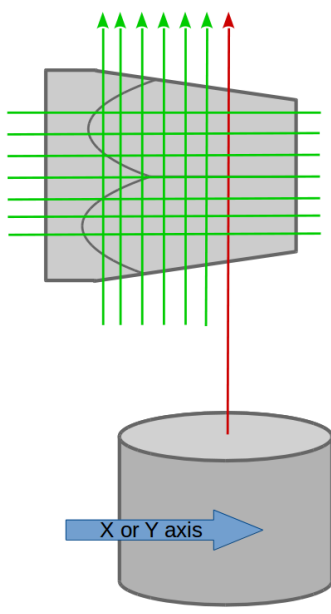

Fig. 4 Simple scheme representing the operations needed to perform the PSCS technique. On the left-hand side the detector is in vertical configuration while on the right-hand side the detector is in horizontal configuration. The red arrows show the direction of the beam during the ongoing data acquisition positions while the green arrows show the direction of the beam at the positions for which data is already acquired.

for a fixed radius along an arc. As the radius is fixed, the rise-time of the main pulse remains constant (there are slight changes due to the fact that charge velocities depend on the germanium crystal orientation). On the other hand the amplitude of the transient signals changes with the interaction position: as the interaction approaches a neighboring segment, the corresponding transient signal (left or right) grows in amplitude.

\section{The Pulse Shape Coincidence Scanning method}

The pulse shape coincidence scan (PSCS) [23] method allows full volume characterization of a given position sensitive detector starting from two sets of data obtained by irradiating the detector with a collimated gamma-ray source. The detector is put at first in vertical configuration (see figure 4), then the collimated source is moved by regular steps in a plane perpendicular to the detector axis. For each position of the collimator all the pulses generated by the detector along the gamma-ray beam are collected and a vertical-beam dataset $\mathcal{V}$ is obtained. The detector is then moved to horizontal configuration by rotating it by $90^{\circ}$ and a horizontal-beam dataset $\mathcal{H}$ is obtained by repeating the same scanning procedure. The positions of the beams of the $\mathcal{V}$ and $\mathcal{H}$ datasets are chosen so that they cross in a three-dimensional reference frame, forming a threedimensional scanning grid.

For a position sensitive detector (such as AGATA) two identical gamma rays that interact in the same point will produce the same signals. If $\mathcal{V}$ and $\mathcal{H}$ datasets are compared, their pulses will differ except where the beams intersect. The similarity between two pulses can be evaluated with the following $\chi^{2}$-like formula

$\chi^{2}=\frac{\sum_{i=0}^{N}\left(v_{i}-h_{i}\right)^{2}}{\sigma \cdot N}$

where $v_{i}$ and $h_{i}$ are the samples of two pulses $\bar{v}$ and $\bar{h}$ coming from the $\mathcal{V}$ and $\mathcal{H}$ datasets, respectively, $\mathrm{N}$ is the total number of samples of each signal (this implies that $\bar{v}$ and $\bar{h}$ are sampled at the same rate) and $\sigma$ is the noise level of the signals. The lower the $\chi^{2}$ value, the most similar are the two compared pulses. An absolute threshold for the $\chi^{2}$ value below which two pulses can be considered identical does not exist. This value, in fact, depends also on the experimental conditions, so that only relative evaluations can be done. In this regard, the main disadvantage of the PSCS technique lies in the fact that multiple interaction events could give rise to small $\chi^{2}$ and appear as fake single interaction events originating at the crossing point. These kind of events may have a negative impact on the final database quality and should be excluded if possible.

\section{Scanning Table}

The IPHC scanning table $[22,30]$ was designed with the aim of performing scans in short scan durations, with good spatial resolution and with variable scanning energies. The table allows to perform a full-volume scan of an AGATA detector in times of the order of 15 days, leading to the creation of a database of $\sim 45000$ points for a $2 \mathrm{~mm}$ scan pitch.

The table setup is shown in figure 3.2 of ref [30]. A metal collimator sits on two motorized perpendicular axes that allow its planar movement in a range of $300 \mathrm{~mm}$ in both $X_{T}$ and $Y_{T}$ directions with a precision of about $10 \mu \mathrm{m}$. Above the collimator, two fixed plates allow the placement of the detector in vertical and horizontal positions. The detector is actually placed in an adjustment frame, which is fixed on the plates by two centering studs. The adjustment frame allows the adjustment between the detector and the table axes, by tilting it using micrometric screws and by freehand rotating it. Finally a laser alignment system is set on the mechanical support of the collimator and is used to keep the relative alignment of the detector when going from the vertical to the horizontal position. 
The collimator, shown in figure 3.4 of [30], consists of a metallic cylindrical block, $189 \mathrm{~mm}$ high with a diameter of $220 \mathrm{~mm}$, composed of iron, lead and tungsten, materials that absorb the gamma rays which are not traveling through the central hole. When scanning an AGATA detector, the distance of the front face of the collimator from the center of the detector in both vertical and horizontal configurations is $54 \mathrm{~mm}$ and $95 \mathrm{~mm}$, respectively. The base of the collimator holds a cylindrical capsule containing the gamma-ray source. The capsule and the overall collimator are coaxial and pierced, along their central axes up to the top of the collimator. Different gamma-ray sources are used for the scans, each one housed in a different capsule. The collimator can be opened by sliding the bottom part and allowing the insertion of the needed source capsule. The central section of the collimator can be mechanically swapped as well and it is possible to choose between three central collimating parts which have diameters of $1.0 \mathrm{~mm}$, $0.5 \mathrm{~mm}$ and $0.2 \mathrm{~mm}$.

Three sources are used for scanning. Each emits gamma rays of different energies that can be used accordingly to their penetration power. An ${ }^{241} \mathrm{Am}$ source, which emits monochromatic gamma rays with an energy of $59.5 \mathrm{keV}$, is used for surface and two-dimensional (2D) scans. A ${ }^{137} \mathrm{Cs}$ source, which emits monochromatic gamma rays with an energy of $661.7 \mathrm{keV}$, is used for deep scanning and for the full-volume characterization (3D) scans. Using the $1 \mathrm{~mm}$ diameter collimator, the ${ }^{137} \mathrm{Cs}$ beam spot diameter at a distance corresponding to the center of an AGATA detector was measured, with a finely pixelated CdTe sensor, to be $1.47 \pm 0.03 \mathrm{~mm}$ and $1.81 \pm 0.03 \mathrm{~mm}$ in vertical and horizontal configuration, respectively. These characteristics are reproduced in the simulations.

Finally a ${ }^{152} E u$ source, which emits gamma rays of several energies in the range of $122 \mathrm{keV}$ to $1408 \mathrm{keV}$, is used to perform several advanced tests on detectors.

\section{Tools of the simulation}

The main tool used for the simulation is the Agata Detector Library (ADL) [10], a software library developed at IKP, Universität zu Köln. The library is used by the AGATA collaboration to calculate the signals generated from the AGATA detectors and build the relative pulse-shapes databases to be used for PSA. ADL uses the theorem of Shockley and Ramo to numerically calculate the pulse shapes.

A simple block scheme of the architecture of ADL is shown in figure 5. Different inputs and parameters should be set in the code before running. At first the

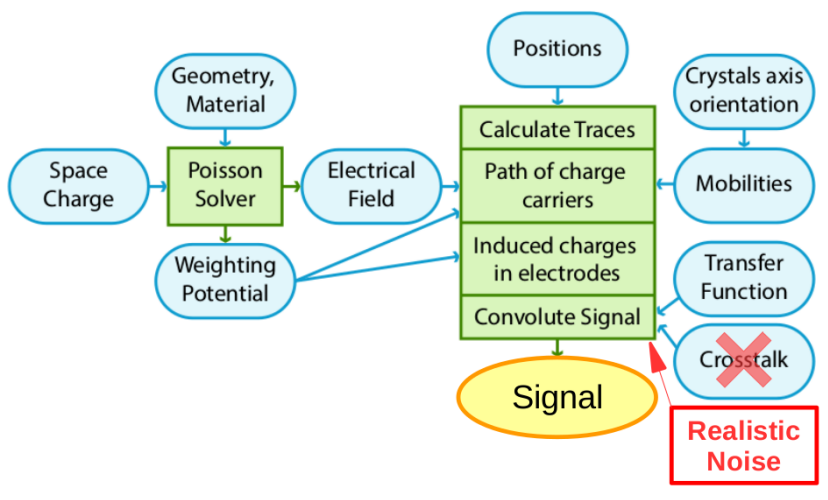

Fig. 5 ADL I/O scheme (adapted from [10]). In blue and red are represented the parameters or inputs to be set in the code while in green are represented the different routines that form the code. In the scheme the crosstalk component is struck off as it is not implemented in the present simulations.

electric field and the weighting potentials for each electrode of the detector are to be defined, as well as the electric field generated by the space charges. These quantities are calculated through a Poisson solver. The ADL software package provides already the weighting potentials, the electric fields and the space charge fields for the 4 different types of AGATA detector (type A, B, C and S). These are calculated with SIMION [34], a software which can be used for discrete calculation of electric fields with complex geometries. The second group of parameters to be set are the mobility parameters for the electrons and holes in the crystal and its axis orientation. For the AGATA detectors these values were determined experimentally in [10].

Once the fields and the mobility parameters are set, it is possible to calculate the pulse shapes generated in an event by giving the positions and the deposited energies as input. These were calculated using the Monte Carlo software Geant4 [35]. Both the detector and collimator were modelized with their exact geometry and materials in order to have an accurate reproduction of the scanning system.

For this work, the pulses calculated by ADL are $1 \mu s$ long, sampled with a rate of $100 \mathrm{MHz}$ (100 samples). In order to have a realistic output, the resulting signals are convoluted with the response function of the preamplifiers. The response function used was taken from the AGAPRO code [36], a software developed by the AGATA collaboration to analyze experimental and simulated data. Realistic noise was added, as well, to the calculated pulses. The characteristics of the noise were extracted from a real S-type detector. Finally, ADL allows the user to convolute the output 

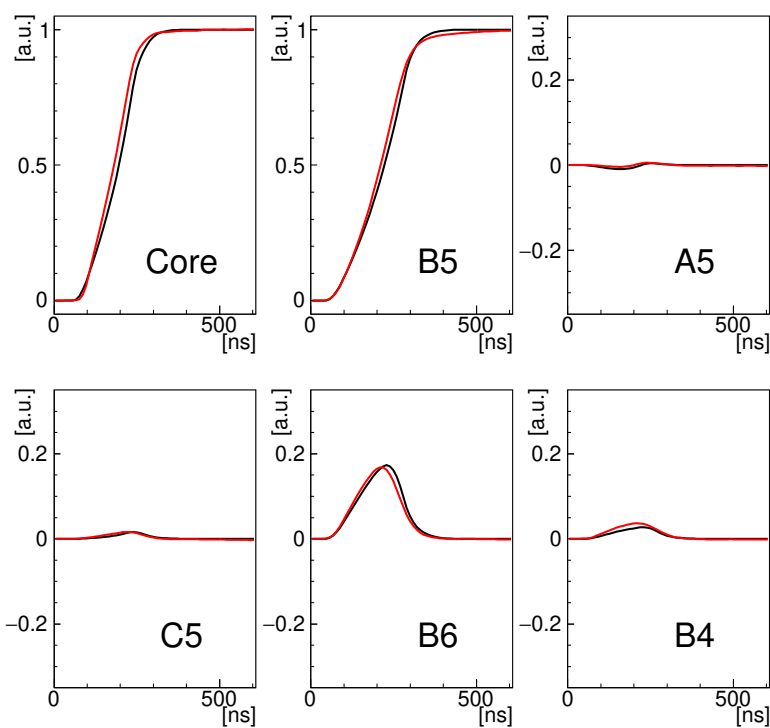

Fig. 6 Comparison between a signal extracted from the measured database of detector S001 (in red) and the corresponding signal calculated with ADL (in black). The first two panels on top show the core and hit segment (B5) signals. A5 and C5 are the right and left neighbor segments, respectively. B6 and $\mathrm{B} 4$ are the top and bottom neighbor segments, respectively.

signal with a crosstalk function, although for this work the crosstalk was not considered.

An example of signals calculated with ADL can be seen in figure 3 . In the example, different signals generated by single interactions in a S-type segmented detector are shown. Figure 6, on the other hand, shows a signal extracted from the database of detector S001, measured with the IPHC scanning table, compared to the corresponding signal calculated with ADL.

\section{Data treatment}

The simulation work-flow is shown in figure 7 . The beam propagation in the detector is simulated with Geant4 and ADL is then used to calculate the pulse shapes generated by the interactions of each gammaray (event). Only the events in which the corresponding gamma ray is fully absorbed in a single segment of the detector are accepted. For each position of the collimator an independent beam file is stored which contains 6000 events. For the full volume scan presented in this work, a total of 2975 beam files are used, 1257 for the vertical configuration and 1718 for the horizontal configuration.

The beam files are processed with a code that implements the pulse-shape comparison algorithm described in section 3 . The overall code design follows the one de-

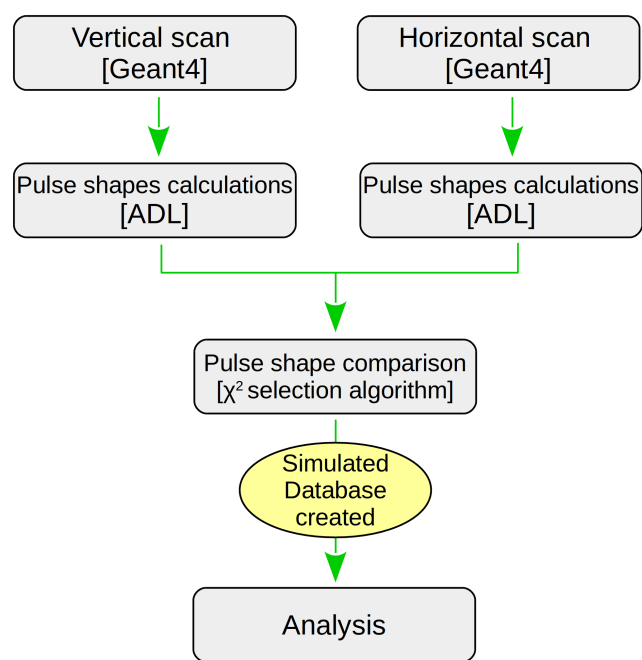

Fig. 7 Work-flow scheme.

scribed in [30]. The comparison between two pulses is performed by calculating the $\chi^{2}$ value with equation

$\chi^{2}=\frac{\sum_{\text {seg } \in \mathcal{M}} \sum_{i=i_{0}}^{i_{f}}\left(v_{\text {seg }, i}-h_{\text {seg }, i}\right)^{2}}{M \cdot\left(i_{f}-i_{0}\right)}$

where seg is the segment index, $\mathcal{M}$ is the $\mathrm{M}$ dimensioned ensemble of segments formed by the Core segment, the hit segment and the neighboring segments of the hit segment (4 segments if the hit segment is in the middle of the detector or 3 segments if the hit segment is at the top or bottom of the detector) and $v_{i}$ and $h_{i}$ are the samples of the vertical and horizontal signals, respectively. Finally, the comparison is performed on a subset of samples in the window $\left[i_{0}, i_{f}\right]$ containing the leading edge of the pulse.

Only the signals produced by events happening in the same segment are compared and this reduces the total amount of couples to compare from $3.6 \cdot 10^{7}$ to a few $10^{6}$. Each pair of events compared is inserted in a $\chi^{2}$ value ordered list and at the end of the procedure only the 400 couples with the lowest $\chi^{2}$ values are selected. Among these, some events may have been selected more than once. The duplicates are discarded from the list before the mean pulse-shape refinement.

The refinement procedure, schematized in figure 8, rejects the most diverging pulses giving a more coherent final selection of events, since the $\chi^{2}$ procedure doesn't insure that the selected pairs are similar within each others. At the beginning of the procedure, the mean pulse of all the events of the final list is calculated. Each pulse is then compared with this mean and the corresponding $\chi^{2}$ is calculated with equation 7 . After that, $5 \%$ of the events associated to the higher $\chi^{2}$ value are rejected and the procedure is repeated for 10 it- 


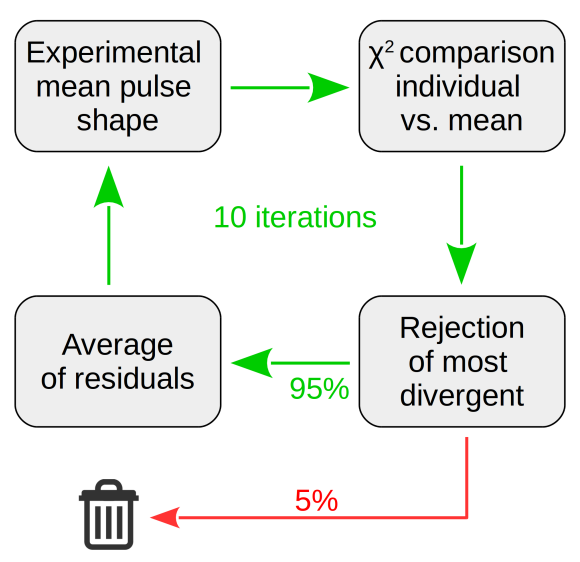

Table 1 Summary table of the simulated scans. All the scans have a pitch of $2 \mathrm{~mm}$ along both axes except the ones in the last two lines, where just 6 points (one per slice) were scanned.

\begin{tabular}{lll}
\hline Description & Energy & Inputs events \\
\hline Full volume scan & $662 \mathrm{keV}$ & $6000^{2}$ \\
Sector scan & $122 \mathrm{keV}$ & $6000^{2}$ \\
Sector scan & $344 \mathrm{keV}$ & $6000^{2}$ \\
Sector scan & $779 \mathrm{keV}$ & $6000^{2}$ \\
Sector scan & $1408 \mathrm{keV}$ & $6000^{2}$ \\
6-points scan & $779 \mathrm{keV}$ & $1000^{2}, 6000^{2}, 60000^{2}$ \\
6-points scan & $1408 \mathrm{keV}$ & $1000^{2}, 6000^{2}, 60000^{2}$ \\
\hline
\end{tabular}

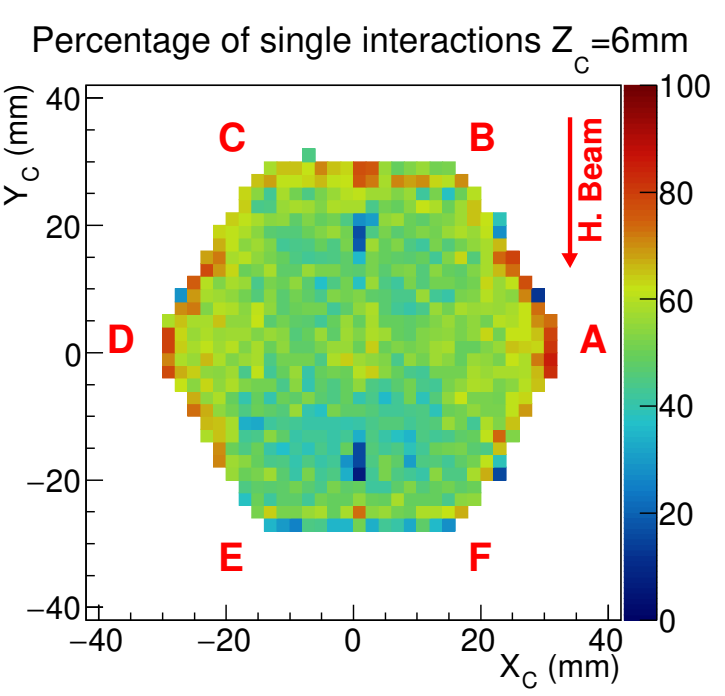

Fig. 9 Reference frame used to present the results of the PSCS technique analysis.

erations. The remaining signals are averaged and the resulting signal will be part of the final database.

The computational times for the selection procedure are of the order of $\sim 7 \mathrm{~s}$ per grid point (on a $2.60 \mathrm{GHz}$ processor machine) leading to a total processing time of $\sim 4.7$ days for a typical simulated scan of an AGATA detector of $\sim 60000$ points which produces (considering the tapering of the detector) a database of $\sim 45000$ points for a S-type detector and $\sim 48000$ for asymmetric crystals.

\section{Three-dimensional scan results with the ${ }^{137} \mathrm{Cs}$ source}

The PSCS technique was tested on a S-type detector by simulating various scans, a list of which is given in table 1. For all the scans the $1 \mathrm{~mm}$ diameter collimator was used. In figure 9 the reference frame in which the results of the analysis will be presented is shown.

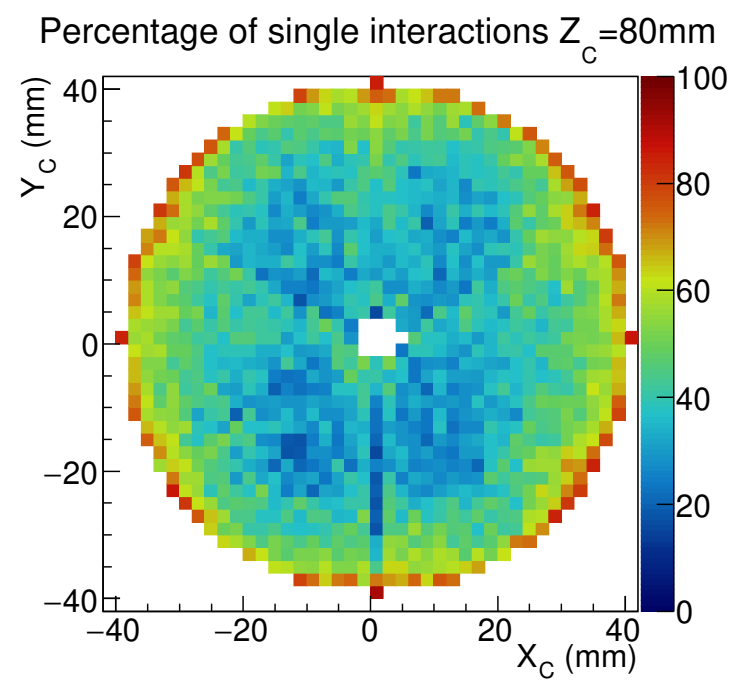

Fig. 10 Percentage of single-interaction events selected by the PSCS technique over the total number of events. The graphs are relative to slices with a thickness of 1 grid unit $\left(2 \mathrm{~mm}\right.$ ) for $Z_{C}=6 \mathrm{~mm}$ (top) and $Z_{C}=80 \mathrm{~mm}$ (bottom). The red arrow in the first panel shows the direction of the beam during the horizontal scan. 


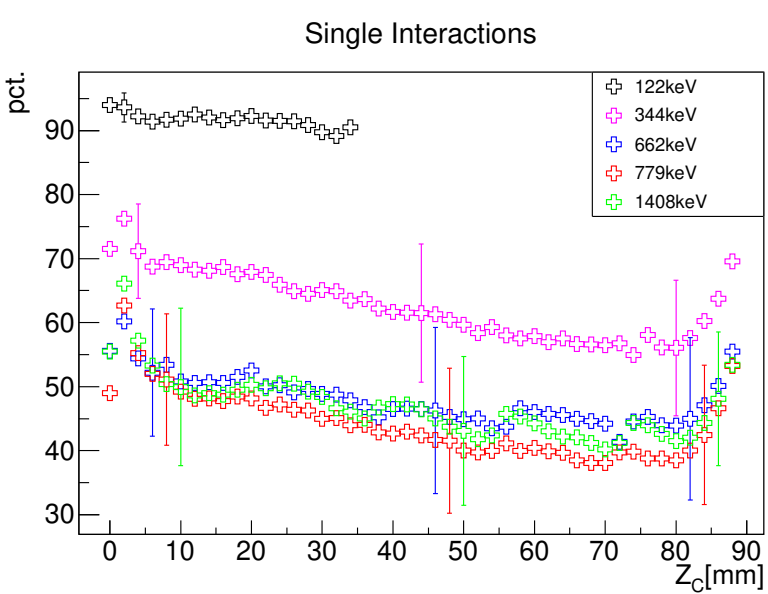

Fig. 11 Percentage (pct.) of selected single-interaction events along the $Z_{C}$ direction for different gamma-ray energies. The bars show the standard deviation for slices at the front, center and back of the detector. Since low energy gamma rays are more likely to be absorbed in the first few centimeters of germanium, no data is available after $Z_{C}=34 \mathrm{~mm}$ for the $122 \mathrm{keV}$ scan.
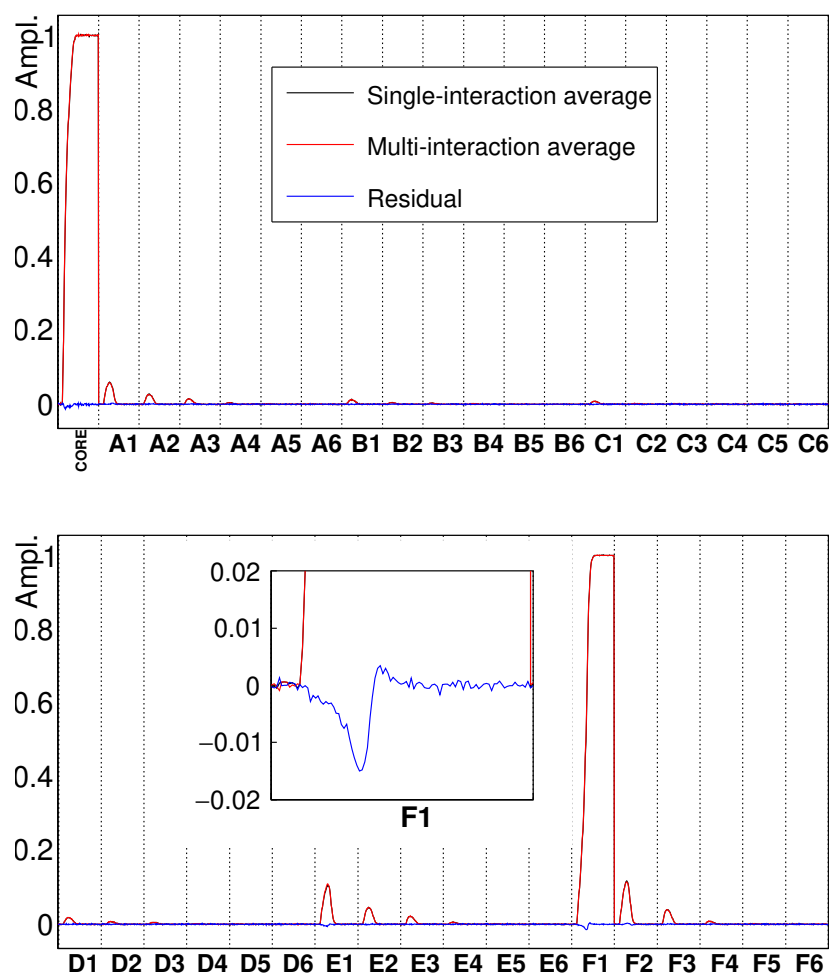

Fig. 12 Example of a supertrace extracted from the database. The supertrace is presented in two panels to fit the text column. The signal is normalized with respect to the main signal amplitude (Ampl.). The graph insert shows a detail of the residual trace for segment F1 (hit segment). Note that the red line is superimposed on the black which is therefore not visible.
At first a full volume ${ }^{137} \mathrm{Cs}$ scan is performed. The first parameter analyzed is the percentage of pulses originating from single-interacting gamma rays (or singleinteraction events) selected by the pulse shape comparison (PSC) algorithm. This value is quite homogeneous across each transversal, $2 \mathrm{~mm}$ thick (with respect to the $Z_{C}$ direction) slice of the database, as shown in figure 10 . However, figure 11 shows that the average value per slice, at $662 \mathrm{keV}$ (blue markers), varies along the depth of the detector, decreasing as $Z_{C}$ increments. The maximum average value of single-interaction events is of about $56 \%$ at the front of the detector while the minimum average value is of around $44 \%$ at the back. The reasons for this trend can be found in two factors. The first one has to do with the geometrical properties of the segments: the active volume of the segments of the S-type detector increases from the front to the back of the crystal and this increases the fraction of selected multi-interaction events among the considered events that release all their energy in a single sector. The second factor concerns the absorption of gamma rays in the germanium: as the beams dips deep in the detector, the available number of events for the $\chi^{2}$ selection is reduced and this leads to a less efficient selection of single-interaction events (the impact of the number of events used for the $\chi^{2}$ selection will be described later in section 9).

A good single-interaction selection is preferred for the building of a database, since multiple interaction events can, in some way, spoil the database. Ideally, the PSC should only select pulses generated by single interactions near the considered database point. This is the case for the Liverpool scanning table [18], which exploits a coincidence scanning technique and reaches a selection of single interactions of $\sim 90 \%$, a simulated value much larger than the ones shown in figures 10 and 11.

For each database point simulated, however, the PSCS technique selects single-interaction and multipleinteraction events which are quite similar. Let's define a supetrace as a compact, continuous representation of all the signals leading edges coming from the 36 segments and the core of the detector (see figure 12). The average supetraces of single-interaction events and multiple-interaction events, for each database point, are calculated as well as their difference called residual supertrace. The maximum absolute value of the residual supertrace (or maximum residual) divided by the amplitude of the main signal, given in percentage, is taken as comparison parameter. The variation of its average value per database slice along the depth of the detector is shown in figure 13 . The average values are quite homogeneous in the volume of the detector $(<5 \%)$ with 


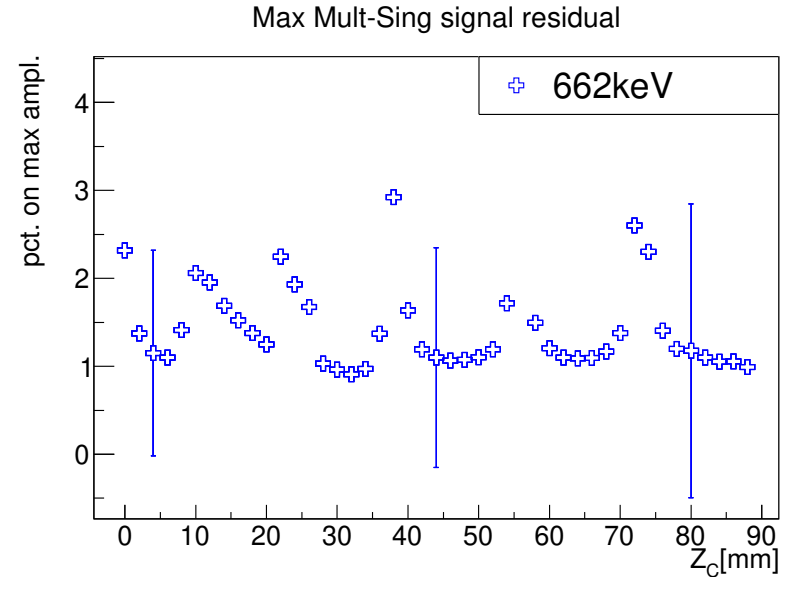

Fig. 13 Maximum residual value, in percent (pct.) with respect to the maximum amplitude of the main signal, between the averaged signals of single-interaction events and the averaged signals of multiple-interaction events along the $Z_{C}$ direction. The bars show the standard deviation for slices at the front, center and back of the detector.

values in the bulk of the segments of $1-1.5 \%$. Some anomalous high values can be registered for points that are towards the segmentation lines due to border effects. In fact, the signals at the borders, especially transient signals, vary very rapidly meaning that a small difference in the interaction position can lead to large differences in the shape of the transient signals.

The similarity between the pulses generated by singleinteraction events and multiple-interaction events can be understood by considering the average distance of the PSCS selected events from the position of their corresponding database point. The values for different transversal database slices at different depths of the detector (see figure 14) show that the average distance is homogeneous across each individual $2 \mathrm{~mm}$ slice. The graph in figure 15 shows a slightly growing trend along $Z_{C}$ for the average values of each slice. At $662 \mathrm{keV}$ (blue markers), the average distance, in fact, has a value of $\sim 2 \mathrm{~mm}$ in the front of the detector and then rises slightly until it reaches $\sim 3 \mathrm{~mm}$ at the back. These values, nevertheless, are comparable to the PSA performances (see table 4 in [1]) and the nominal position resolution of an AGATA detector.

Finally, a second reason that explains the similarity between the selected single-interaction events and multiple-interaction events pulses is found by considering, for multiple-interaction events, the energy released by the furthest interaction from the first interaction point as a function of the distance between these two points. The examples in figure 16 show that generally the furthest interaction releases the least energy and thus contributes less to the signal formation. In other

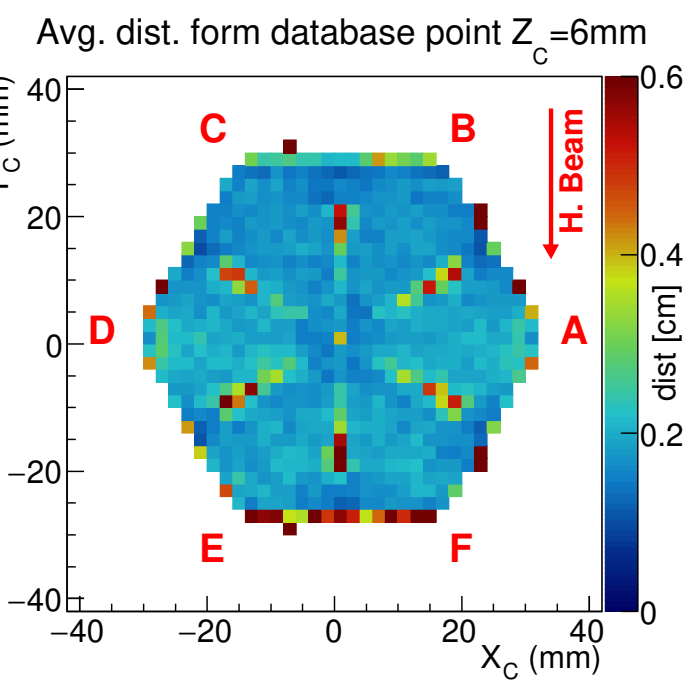

Avg. dist. form database point $Z_{C}=80 \mathrm{~mm}$

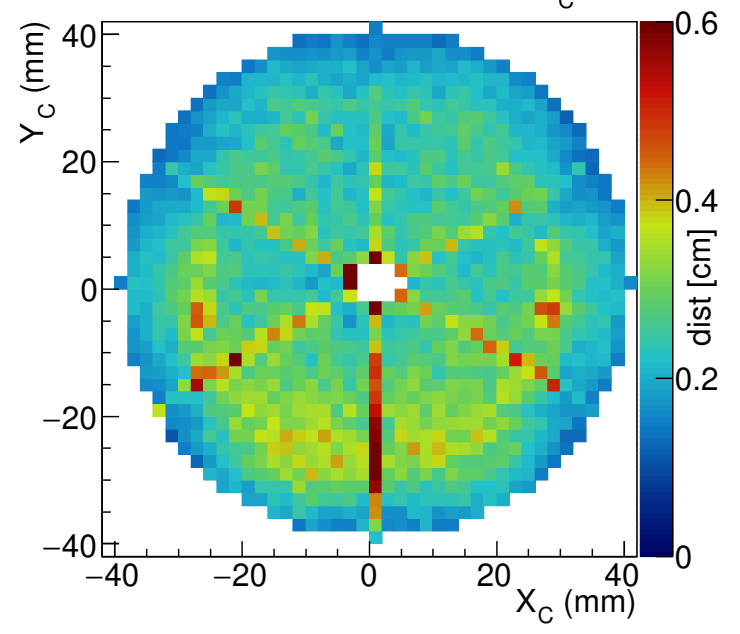

Fig. 14 Average distance (in $\mathrm{cm}$ ) of the interactions of the PSCS selected events from the position of their database point. The graphs are relative to slices with a thickness of 1 grid unit $\left(2 \mathrm{~mm}\right.$ ) for $Z_{C}=6 \mathrm{~mm}$ (top) and $Z_{C}=80 \mathrm{~mm}$ (bottom). The red arrow in the first panel shows the direction of the beam during the horizontal scan.

words, the bulk of the signal is formed near the position of the first interaction.

In the next two sections the PSCS technique will be further tested with scans performed with different gamma-ray energies and different number of pulses used as input of the $\chi^{2}$ selection.

\section{Impact of the energy on the PSCS technique performance}

As a further test of the PSCS technique, the impact of the gamma-ray energy used for the scan was evalu- 


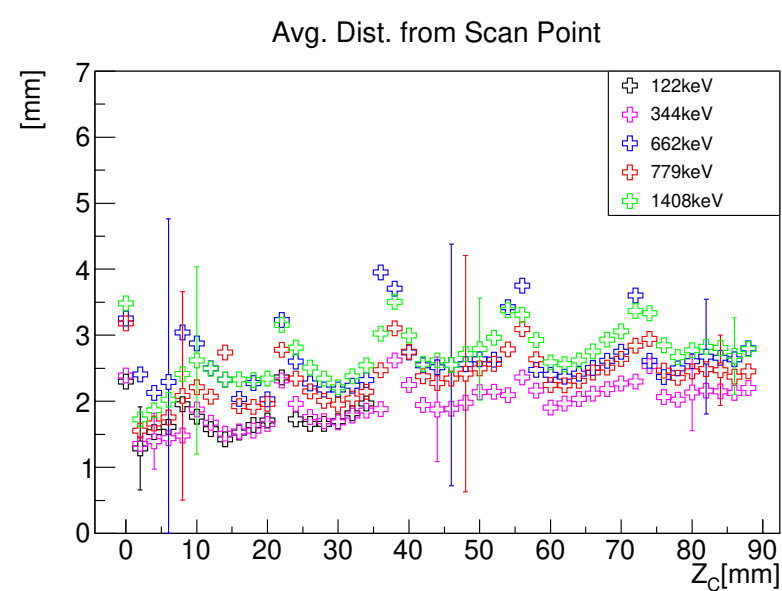

Fig. 15 Average distance of interactions of the PSCS selected events from the position of their database point along the $Z_{C}$ direction for different gamma-ray energies. The bars show the standard deviation for slices at the front, center and back of the detector.

ated. Four simulated partial scans (only one sector of the detector was scanned) were performed with energies of $122 \mathrm{keV}, 344 \mathrm{keV}, 779 \mathrm{keV}$ and $1408 \mathrm{keV}$ which correspond to four of the various gamma-ray energies emitted by a ${ }^{152} \mathrm{Eu}$ source. The parameters considered for the present analysis are:

- the percentage of selected single-interaction events;

- the average distance of the selected events from the position of their corresponding database point.

Figure 11 shows the percentage of selected single-interaction events at different depths of the detector for the different scanning energies. The graphs show that data series follow the same trend as already discussed for $662 \mathrm{keV}$ in 7. However, the gamma-ray energy has an impact on the PSCS results. For low energies $(122 \mathrm{keV}$ and $344 \mathrm{keV}$ ), the fraction of selected single-interaction events is sensibly higher with respect to the energies above $500 \mathrm{keV}$. This result is due to the fact that gamma rays of lower energies rays are more likely to undergo photo-absorption. This means that for each pool of events for a given scanned position, the fraction of single-interaction events is already larger before the $\chi^{2}$ selection than for higher energies.

The average distance of the selected events from the position of their corresponding database point exhibits a tiny energy dependence, the higher energy gammarays having the largest average distance values (figure 15). However, the overall values are quite comparable and range between $1 \mathrm{~mm}$ and $3 \mathrm{~mm}$. It is noteworthy that at positions around $Z_{C}=14 \mathrm{~mm}, 22 \mathrm{~mm}, 36 \mathrm{~mm}$, $56 \mathrm{~mm}$, and $74 \mathrm{~mm}$ the average distance peaks at $\sim$ $4 \mathrm{~mm}$ due to the presence of the segment boundaries (see figure 17).
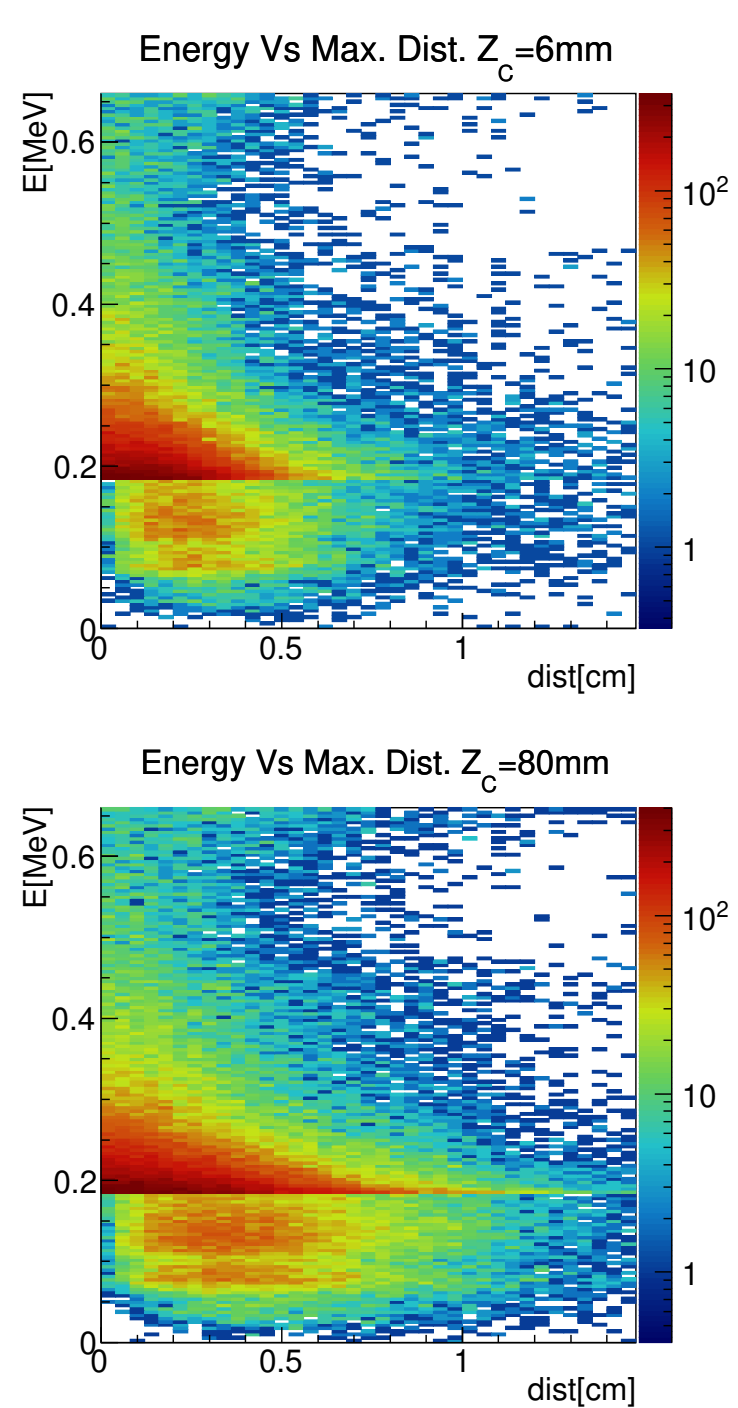

Fig. 16 For multiple-interaction events, energy released by the furthest interaction from the first interaction point as a function of the distance between these two points. Each graph gathers the interactions of all the points for a slice of the database with a thickness of 1 grid unit $(2 \mathrm{~mm})$ for $Z_{C}=6 \mathrm{~mm}$ (top) and $Z_{C}=80 \mathrm{~mm}$ (bottom).

\section{Impact of the statistics on the PSCS technique performance}

The impact on the PSCS technique of the number of pulses used as input for the $\chi^{2}$ selection is evaluated. A scan of six points along the $Z_{C}$ direction was performed, as shown in figure 17 , so that each point is roughly in the center of each segment of a sector (sector D). The $\chi^{2}$ selection was performed by comparing $1000 \times$ 1000 (low statistics), $6000 \times 6000$ (regular simulation statistics) and $60000 \times 60000$ (high statistics) pulses for each point of the scan. Two gamma-ray energies were used, $779 \mathrm{keV}$ and $1408 \mathrm{keV}$ and, as in the previous 


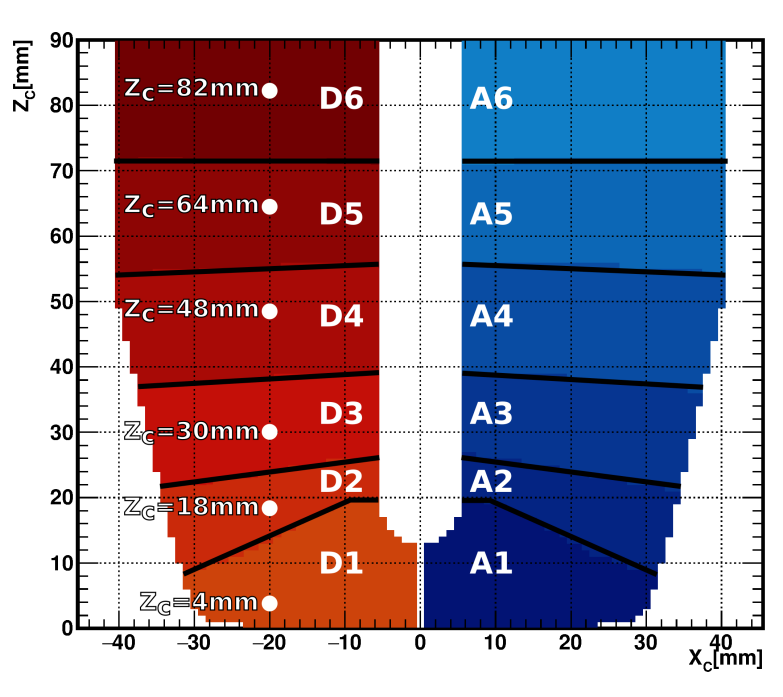

Fig. 17 Separation surface of the segments due to the electric field. In the picture the central section of the detector is shown. The $Z_{C}$ positions of the six scanned points at $X_{C}=-20 \mathrm{~mm}$ and $Y_{C}=0 \mathrm{~mm}$ analyzed in section 9 are also shown.
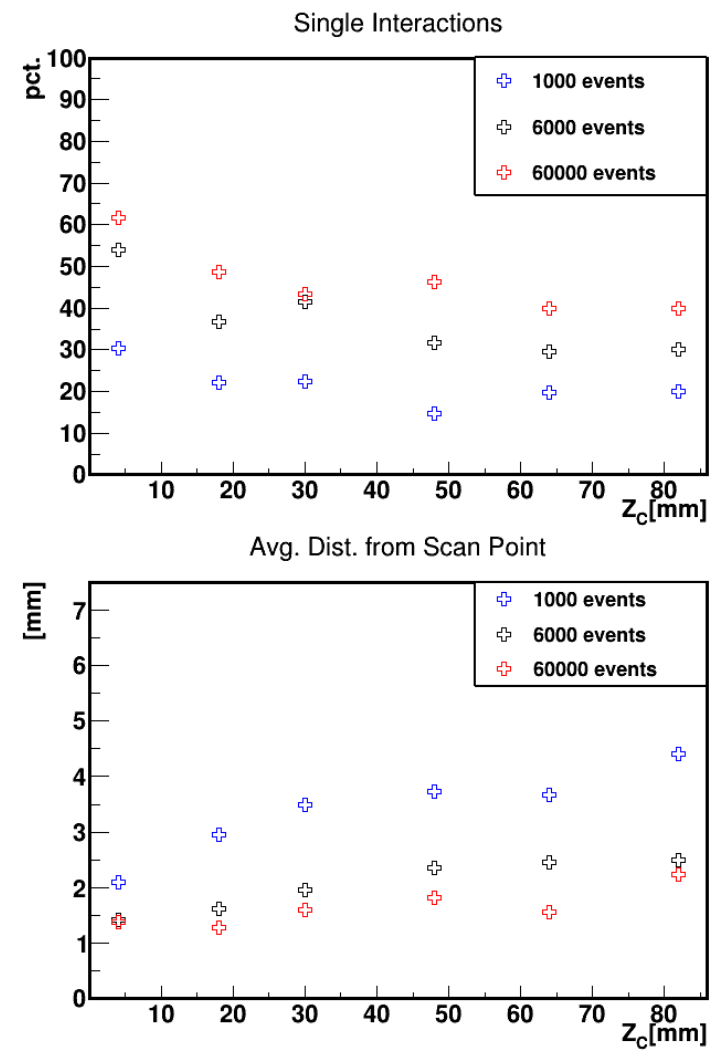

Fig. 18 Values of the two considered parameters along the $Z_{C}$ direction for the $779 \mathrm{keV}$ scan. In the top panel: percentage of selected single-interaction events. In the bottom panel: average distance of the selected events from the position of their corresponding database point.
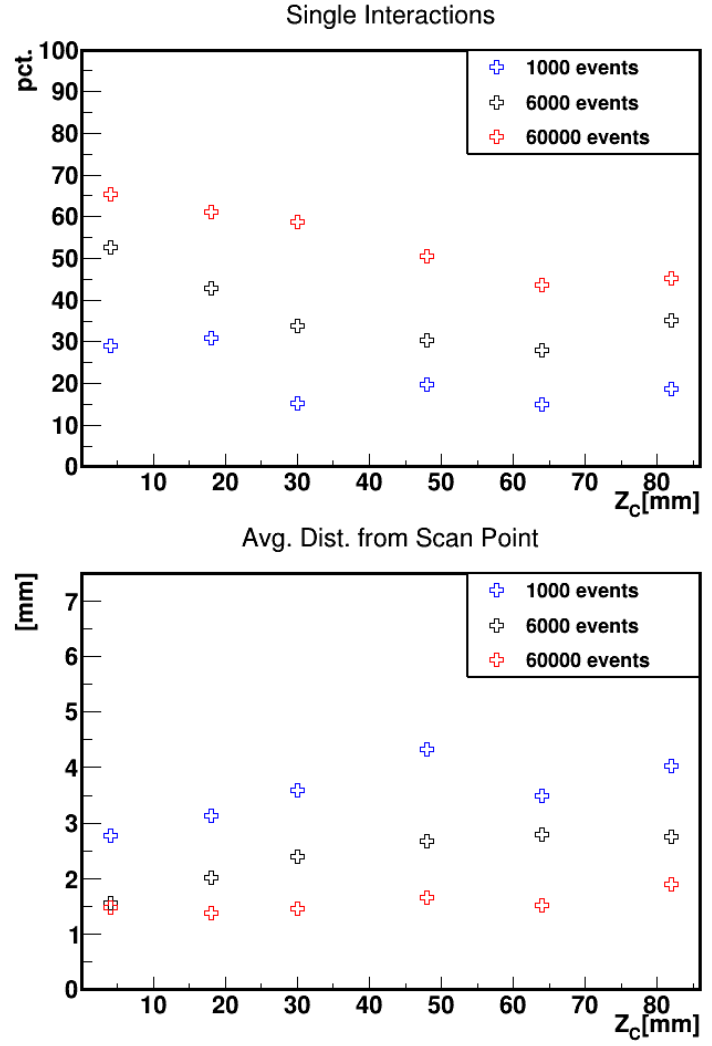

Fig. 19 Values of the two considered parameters along the $Z_{C}$ direction for the $1408 \mathrm{keV}$ scan. $\mathrm{n}$ the top panel: percentage of selected single-interaction events. In the bottom panel: average distance of the selected events from the position of their corresponding database point.

paragraph, the two main parameters considered for this analysis are:

- the percentage of selected single-interaction events;

- the average distance of the selected events from the position of their corresponding database point.

The results, plotted in figure 18 (19) for the $779 \mathrm{keV}$ $(1408 \mathrm{keV})$ gamma-ray energy scans show that statistics plays an important role in the PSCS selection. In fact, although the trends of the data series of the two parameters previously listed is identical, their values are noticeably affected.

The percentage of selected single-interaction events increases substantially and the average distance of the selected events from the position of their corresponding database point decreases with the number of pulses used for the $\chi^{2}$ selection. This last phenomenon is foreseen in the bottom panel of figure 14 where a general, slight increase of the average distance from the database point is visible in correspondence of sectors $\mathrm{E}$ and $\mathrm{F}$ $\left(Y_{C}<0\right)$. In fact, to reach this area the beam traverses the largest thickness of germanium in both the vertical and horizontal (see the red arrow in the top panel) 
scans, getting absorbed in the process. As a result, the statistics for the $\chi^{2}$ selection is affected, leading to the effects described in this section. In conclusion, it is clear that for these two parameters the PSCS technique performs better when a higher number of pulses is used for the $\chi^{2}$ selection. As a further analysis it would be worth to extend the range of the test to even lower statistics (few hundreds of events) and higher statistics (orders of magnitude $>10^{4}$ ).

\section{Conclusions and Perspectives}

A study of the PSCS technique implemented at IPHC to scan germanium detectors in $2 \mathrm{D}$ and $3 \mathrm{D}$ was performed via simulations and the results were discussed. This work was aimed to characterize the accuracy and the critical points of the scanning procedure in order to estimate whether the PSCS technique is able to provide full crystal volume, experimental, pulse-shape databases. A simulation of a full volume scan of a S-type AGATA detector was performed with a gamma-ray beam energy of $662 \mathrm{keV}$ ( ${ }^{137} \mathrm{Cs}$ source) using the GEANT4 and ADL softwares. The pulse shapes generated were convoluted with realistic noise and the response functions of the preamplifiers.

The first parameter extracted from the analysis was the percentage of the selected pulses generated by a single interaction. The values obtained indicated a percentage of single interactions that ranges, in average between $55 \%$ at the front of the detector and $45 \%$ at its back, which is lower than expected.

The influence of multiple interaction events on the average pulse shape at each point of the scan grid was investigated. The analysis showed that the many interaction points of multiple interaction events occur relatively close to each other and that the average value of their distance from the corresponding scan grid point is around $2 \mathrm{~mm}$. Moreover it was observed that the multiple interaction pulses selected by the PSCS technique are similar to those of single interactions. In addition, the simulations showed that for a multiple scattering event, the energy deposition of the interaction point furthest from the first interaction decreases as the distance between these two points increases. Typically, the largest energy release occurs in the vicinity of the first interaction where most of the signal is generated. All these elements explained the similarity between the pulses generated by single and multiple interaction events which lead us to conclude that the multiple interactions pulses selected by the PSCS technique do not contaminate the database.

The impact of the gamma-ray energy on the extracted pulse shapes was examined at $122 \mathrm{keV}, 344 \mathrm{keV}$,
$779 \mathrm{keV}$ and $1408 \mathrm{keV}$ (europium source energies). The analysis showed that the energy of the gamma-ray beam impacted significantly only the percentage of selected single interaction events, enhancing this value for low energies gamma rays. The average distance of the selected events from their corresponding database point, instead, showed only a minimal energy dependence with values that are overall comparable within the data series and with the results obtained for the $662 \mathrm{keV}$ scan.

The impact of the input statistics was also analyzed using different numbers of input events to be compared with the $\chi^{2}$ selection algorithm for both the vertical and the horizontal datasets. The input statistics proved to have a larger impact than the beam energy, the PSCS technique performing better with higher input statistics. The overall results indicate that the PSCS technique applied to an AGATA unit detector is reliable.

This work shows that further studies can be performed. Different values of the power in equation 6 can be used as in [37]. The $\chi$-like formula would then become $\chi^{\alpha}$ where $\alpha$ is a value that can be optimized in order to maximize the percentage of selected single interactions and minimize the average distance of the interaction points from the respective database position. Another way to improve the $\chi^{2}$ selection can be the use of weights for the transient signals to give them more or less impact on the database construction. This option was preliminarily tested in [30] and a more systematic study with extensive simulations can be done. Scans with a finer scanning pitch and smaller diameter of the collimator can be simulated. These scans can lead to improvements in the PSCS selection especially near the segmentation surfaces where it is critical to have a finer resolution for the database since in these areas the transient signals change very rapidly for small changes in the position.

Finally, real scans with an ${ }^{152} E u$ source have been performed recently for the first time and the results will be presented in an upcoming publication [24, 38]. These measurements will likely give hints to further improve the PSCS technique.

Acknowledgements This work was partially supported by the MIRION Technologies Canberra Company under the contract No 152251.

\section{References}

1. S. Akkoyun, et al. AGATA - Advanced GAmma Tracking Array. Nuclear Instruments and Methods in Physics Research Section A: Accelerators, Spectrometers, Detectors and Associated Equipment, 668:26 - 58, 2012. 
2. R. Venturelli. and D. Bazzaco. Adaptive grid search as pulse shape analysis algorithm for gammatracking and results. LNL Annual Report, page 220, 2004.

3. A. Lopez-Martens, et al. Gamma-ray tracking algorithms: a comparison. Nuclear Instruments and Methods in Physics Research Section A: Accelerators, Spectrometers, Detectors and Associated Equipment, 533(3):454 - 466, 2004.

4. G. Suliman and D. Bucurescu. Fuzzy clustering algorithm for gamma ray tracking in segmented detectors. Romanian Reports in Physics, 62:27-36, 2010 .

5. F. Didierjean, et al. The Deterministic Annealing Filter: A new clustering method for gammaray tracking algorithms. Nuclear Instruments and Methods in Physics Research Section A: Accelerators, Spectrometers, Detectors and Associated Equipment, 615:188-200, 2010.

6. S. Tashenov and J. Gerl. Tango - new tracking algorithm for gamma-rays. Nuclear Instruments and Methods in Physics Research Section A Accelerators Spectrometers Detectors and Associated Equipment, 622:592-601, 2010.

7. F.A. Beck. EUROBALL: Large gamma ray spectrometers through european collaborations. Progress in Particle and Nuclear Physics, 28:443 - 461, 1992.

8. J. Simpson. The EUROBALL spectrometer. Zeitschrift für Physik A Hadrons and Nuclei, 358(2):139-143, 1997.

9. C.W. Beausang, et al. Measurements on prototype Ge and BGO detectors for the EUROGAM array. Nuclear Instruments and Methods in Physics Research Section A: Accelerators, Spectrometers, Detectors and Associated Equipment, 313(1):37 - 49, 1992.

10. B. Bruyneel, et al. Pulse shape analysis and position determination in segmented HPGe detectors: The AGATA detector library. The European Physical Journal A, 52(3):70-80, 2016.

11. L. Mihailescu. Principles and Methods for gamma ray tracking with large volume Germanium Detectors. Ph.D. thesis, University of Bonn, 2000.

12. L. Mihailescu, et al. The influence of anisotropic electron drift velocity on the signal shapes of closedend HPGe detectors. Nucl. Instrum. Meth. A, 447:350-360, 2000.

13. P. Medina, et al. A simple method for the characterization of HPGe detectors. Conference Record IEEE Instrumentation and Measurement Technology Conference, 3:1828 - 1832 Vol.3, 2004.
14. M. Schlarb, et al. Pulse shape analysis for gamma-ray tracking (Part I): Pulse shape simulation with JASS. The European Physical Journal A, 47(10):132-155, 2011.

15. I. Mateu, et al. Simulation of the charge collection and signal response of a HPGe double sided strip detector using MGS. Nuclear Instruments and Methods in Physics Research A, 735:574-583, 2014.

16. F. Recchia, et al. Position resolution of the prototype AGATA triple-cluster detector from an inbeam experiment. Nuclear Instruments and Methods in Physics Research Section A: Accelerators, Spectrometers, Detectors and Associated Equipment, 604(3):555 - 562, 2009.

17. P.-A. Soederstroem, et al. Interaction position resolution simulations and in-beam measurements of the AGATA HPGe detectors. Nuclear Instruments and Methods in Physics Research Section A: Accelerators, Spectrometers, Detectors and Associated Equipment, 638(1):96 - 109, 2011.

18. Matthew Dimmock, et al. Validation of Pulse Shape Simulations for an AGATA prototype detector. Nuclear Science, IEEE Transactions on, $56: 2415-2425,2009$.

19. T.M.H. Ha, et al. New setup for the characterisation of the AGATA detectors. Nuclear Instruments and Methods in Physics Research Section A: Accelerators, Spectrometers, Detectors and Associated Equipment, 697:123 - 132, 2013.

20. N. Goel, et al. Spatial calibration via imaging techniques of a novel scanning system for the pulse shape characterisation of position sensitive HPGe detectors. Nuclear Instruments and Methods in Physics Research Section A: Accelerators, Spectrometers, Detectors and Associated Equipment, 652(1):591 - 594, 2011. Symposium on Radiation Measurements and Applications (SORMA) XII 2010.

21. A. Hernandez-Prieto, et al. Study of accuracy in the position determination with SALSA, a gammascanning system for the characterization of segmented HPGe detectors. Nuclear Instruments and Methods in Physics Research Section A: Accelerators, Spectrometers, Detectors and Associated Equipment, 823:98 - 106, 2016.

22. M.H. Sigward, et al. Pulse-Shape Comparison Scan of germanium detectors using the IPHC scanning table. To be published.

23. F.C.L. Crespi, et al. A novel technique for the characterization of a HPGe detector response based on pulse shape comparison. Nuclear Instruments and Methods in Physics Research Section A: Accelerators, Spectrometers, Detectors and Associated 
Equipment, 593(3):440 - 447, 2008.

24. B. De Canditiis. 3D characterization of multisegmented HPGe detectors. Simulation and validation of the PSCS technique and its application for different energies with a ${ }^{152} \mathrm{Eu}$ source. Ph.D. thesis, University of Strasbourg, 2020.

25. J. Simpson, et al. AGATA Technical Design Report. Technical report, 2008.

26. J. Eberth, et al. Encapsulated Ge detectors: Development and first tests. Nuclear Instruments and Methods in Physics Research Section A: Accelerators, Spectrometers, Detectors and Associated Equipment, 369(1):135 - 140, 1996.

27. A. Pullia, et al. An advanced preamplifier for highly segmented germanium detectors. IEEE Transactions on Nuclear Science, 53(5):2869-2875, 2006.

28. Francesca Zocca, et al. A time-over-threshold technique for wide dynamic range gamma-ray spectroscopy with the AGATA detector. Nuclear Science, IEEE Transactions on, 56:2384 - 2391, 2009.

29. G. Pascovici, et al. Low noise, dual gain preamplifier with built in spectroscopic pulser for highly segmented high-purity germanium detectors. WSEAS Trans. Cir. and Sys., 7(6):470-481, 2008.

30. M. Ginsz. Characterization of high-purity, multi-segmented germanium detectors. Ph.D. thesis, University of Strasbourg, 2015. https://publication-theses. unistra.fr/ public/theses_doctorat/2015/Ginsz_Michael_ 2015_ED182.pdf.

31. W. Shockley. Currents to conductors induced by a moving point charge. Journal of Applied Physics, 9:635-636, 1938.

32. S. Ramo. Currents induced by electron motion. Proceedings of the I.R.E., 27:584-585, 1939.

33. Zhong He. Review of the Shockley-Ramo theorem and its application in semiconductor gammaray detectors. Nuclear Instruments and Methods in Physics Research Section A: Accelerators, Spectrometers, Detectors and Associated Equipment, 463(1):250 - 267, 2001.

34. SIMION https://simion.com/.

35. Geant4 https://geant4.web.cern.ch/.

36. AGAPRO section on the GSI forum https://forum.gsi.de/index.php?t=thread\& frm_id=219\&rid=0.

37. L. Lewandowski, et al. Pulse-Shape Analysis and position resolution in highly segmented HPGe AGATA detectors. The European Physical Journal A, 55(5):81-93, 2019.

38. B. De Canditiis, et al. To be published. 